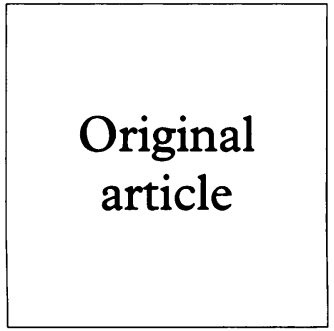

\title{
Diagnosis of neurosyphilis: appraisal of clinical caseload
}

\author{
C A Rodgers, S Murphy
}

Objectives: To review the management of a cohort of patients with positive treponemal serology and psychiatric and/or neurological disorders.

Methods: A retrospective case note review of 172 patients with positive treponemal serology attending the Patrick Clement's Clinic, Central Middlesex Hospital between December 1990 and November 1995 was performed.

Results: 101 men and 71 women were new attenders diagnosed with positive treponemal serology. A neurological problem was identified in 27 patients (12 women and 15 men) with psychiatric and/or neurological disorders, of whom 20 (six women and 14 men) underwent investigation of the cerebrospinal fluid (CSF). With the medical history and results of CSF-RPR and FTA tests, white cell count (WCC), and total protein level in the CSF, 10 patients (eight men and two women) were diagnosed with likely neurosyphilis and 17 with neurological disorders not thought to be caused by syphilis. The clinical features in those having neurosyphilis were sensorineural hearing loss $(n=5)$ and tabes dorsalis $(n=5)$. In the seven patients diagnosed with neurosyphilis who underwent CSF examination one patient had a reactive CSF-FTA, elevated protein, and elevated WCC; one patient had a reactive CSF-FTA and RPR with elevated protein; the total protein only was elevated in three cases and the WCC elevated in one case. Nine of the 10 patients with neurosyphilis received adequate neurosyphilitic treatment; one patient was lost to follow up.

Conclusions: The management of patients with positive treponemal serology and psychiatric and/or neurological disorders was consistent. Patients with suspected neurosyphilis or patients with neurological signs compatible with neurosyphilis (who did not undergo CSF examination) were treated with adequate neurosyphilitic therapy.

(Genitourin Med 1997;73:528-532)

Keywords: neurosyphilis, management

\section{Introduction}

Syphilis is now a rare disease. In the pre-penicillin era the incidence of infectious syphilis in England and Wales was 46 cases per $100000 .^{1}$ By 1994 the incidence of new cases of infectious syphilis had declined to 0.6 cases per 100000 and the total number of cases with positive treponemal serology treated in England and Wales was $1328 .^{2}$ Recent epidemiological patterns show an increase in the prevalence of syphilis in the former USSR and third world countries. ${ }^{34}$

In the past the diagnosis of syphilis was made on clinical findings and confirmed by serological testing. ${ }^{5}$ Diagnosis of syphilis nowadays is frequently only made on serological testing. No serological test can distinguish between the non-venereal treponematoses (yaws, endemic syphilis, and pinta) and venereal syphilis. ${ }^{67}$ The diagnosis of syphilis is supported by serological testing and established by CSF examination. The diagnostic difficulty of differentiating between latent venereal and non-venereal syphilis is increased when patients originate from areas where nonvenereal treponematoses are endemic. In neurosyphilis the problems are further compounded because the signs of neurosyphilis are not pathognomonic and often overlap with signs of other diseases. ${ }^{8}$

Neurosyphilis can develop as a manifestation of both early and late syphilis. Neuro- syphilitic syndromes in secondary syphilis include meningitis and cranial nerve palsies. In tertiary syphilis three distinct clinical syndromes have been described; meningovascular syphilis, parenchymous syphilis, and tabes dorsalis. CNS gummata may also develop. ${ }^{9}$ Traditional CSF criteria for central nervous system involvement in syphilis are a positive Venereal Disease Research Laboratory reaction (VDRL), pleocytosis, and raised protein content (WHO 1982; CDC 1985). ${ }^{111}$ A nonreactive VDRL or raised protein reagin (RPR) test on CSF does not exclude neurosyphilis in a patient having clinical evidence of neurosyphilis ${ }^{12}$ and normal CSF parameters have also been reported in such patients. ${ }^{13}$ The more sensitive Treponema pallidum haemagglutination assay (TPHA) and fluorescent treponemal antibody absorption (FTA-ABS) test in the CSF are not fully reliable. ${ }^{1415}$

The London Borough of Brent is the most multiethnic borough in the United Kingdom ${ }^{16}$ and the management of positive treponemal serology in new attenders to the local genitourinary medicine clinic serving this population, encompasses the diagnostic challenges highlighted above.

The present study was performed to review the diagnosis of neurosyphilis in patients with positive treponemal serology and psychiatric and/or neurological disorders and their subsequent management. 
Table 1 Serum analysis of cohort

\begin{tabular}{lllcc}
\hline Serum analysis & & & Male & Female \\
\hline TPHA & RPR & FTA-Ab & & \\
+ & - & + & 46 & 44 \\
+ & + & + & 49 & 27 \\
+ & - & - & 4 & 1 \\
- & - & + & $1^{\star}$ & $1^{\star}$ \\
- & + & + & $11^{\star}$ & 0 \\
& Total & & $101^{1}$ & 71 \\
\hline
\end{tabular}

TPHA $=$ Treponema pallidum haemagglutination; $\mathrm{RPR}=$ rapid plasmin reagin; FTA-Ab = fluorescent treponema absorption antibody; $\star_{\text {false positive results. }}$

\section{Materials and method}

A retrospective case note review of all new attenders to the Department of Genito-urinary Medicine with positive treponemal serology between December 1990 and November 1995 was performed. Data were extracted on age; country of origin; source of referral; history of yaws, venereal syphilis, genital ulceration and skin rash compatible with secondary syphilis; history of treatment; psychiatric and/or neurological disorder; serology and CSF analysis; final diagnosis and treatment received.

\section{Results}

In all, 101 men and 71 women were new attenders with positive treponemal serology. Medical records of all patients were available for review. The median age of the men was 50 (age range 20-74) and that of the women 38 (age range 19-84).Twelve per cent were West European, $2 \%$ East European, 44\% AfroCaribbean, $29 \%$ African, $8 \%$ Asian, and 2\% South American. Three patients were HIV positive.

Seventy one patients $(41 \%)$ were self referred to the Department of Genito-urinary Medicine, 24 (14\%) attended through partner notification of an STD, $25(14 \%)$ were general practitioner referrals, $17(10 \%)$ were referred as a result of antenatal screening, $13(8 \%)$ were referred from the Department of Ear, Nose and Throat Surgery, $10(6 \%)$ from the Department of General Medicine, and eight $(5 \%)$ by psychiatry. The remaining four patients $(2 \%)$ were referred by the Blood Transfusion Service, the Department of Surgery, a family planning clinic, and another GUM clinic.
Table 1 shows the reactivity of the serum RPR, TPHA, and FTA-ABS and the figure the final diagnosis in the 145 patients without a neurological problem. Neurological examination was performed on 27 patients (12 women and $15 \mathrm{men}$ ) with psychiatric and/or neurological disorders, of whom 20 (six women and 14 men) underwent investigation of the cerebrospinal fluid (CSF). A CSF leucocyte count of $4 \times 10^{6} / 1$ and CSF total protein of $50 \mathrm{mg} / \mathrm{dl}$ was considered significant (WHO criteria).

With the medical history and results of CSF-RPR and FTA tests, white cell count (WCC) and total protein level in the CSF, 10 patients (eight men and two women) were diagnosed with likely neurosyphilis (Table 2).The clinical features in those having neurosyphilis were sensorineural hearing loss (n $=5$ ) and tabes dorsalis $(n=5)$. One patient with tabes dorsalis had an unsuccessful attempt at CSF examination and the diagnosis was made on clinical signs alone (case 3 ) and a second patient with clinically diagnostic tabes dorsalis had a normal CSF examination (case 10). One patient with sensorineural hearing loss declined CSF examination (case 2). In the seven patients diagnosed with neurosyphilis who underwent CSF examination one patient had a reactive CSF-FTA, elevated protein, and elevated WCC (case 4); one patient had a reactive CSF-FTA and RPR with elevated protein (case 6); the total protein only was elevated in cases 5,8 , and $9(n=3)$ and the WCC elevated in one (case 7). Nine of the 10 patients with neurosyphilis received adequate neurosyphilitic treatment; one patient was lost to follow up (case 1). Four patients gave a history of yaws for which two had previously received treatment. No patient gave a history of venereal syphilis.

Seventeen patients had neurological disorders not thought to be due to syphilis, of whom 10 had a normal CSF examination and seven refused the procedure (Tables 3 and 4). Of the 10 patients with normal CSF factors seven were diagnosed with latent non-venereal syphilis and concomitant hypertensive cerebrovascular disease (cases 2, 5, and 6), Ménière's disease (case 7), sensorineural hearing loss (case 8), diabetes mellitus (case 1), and mild memory impairment (case 10). All
Diagnosis in cohort of patients with positive treponemal serology without neurologica disease $(n=145)$.

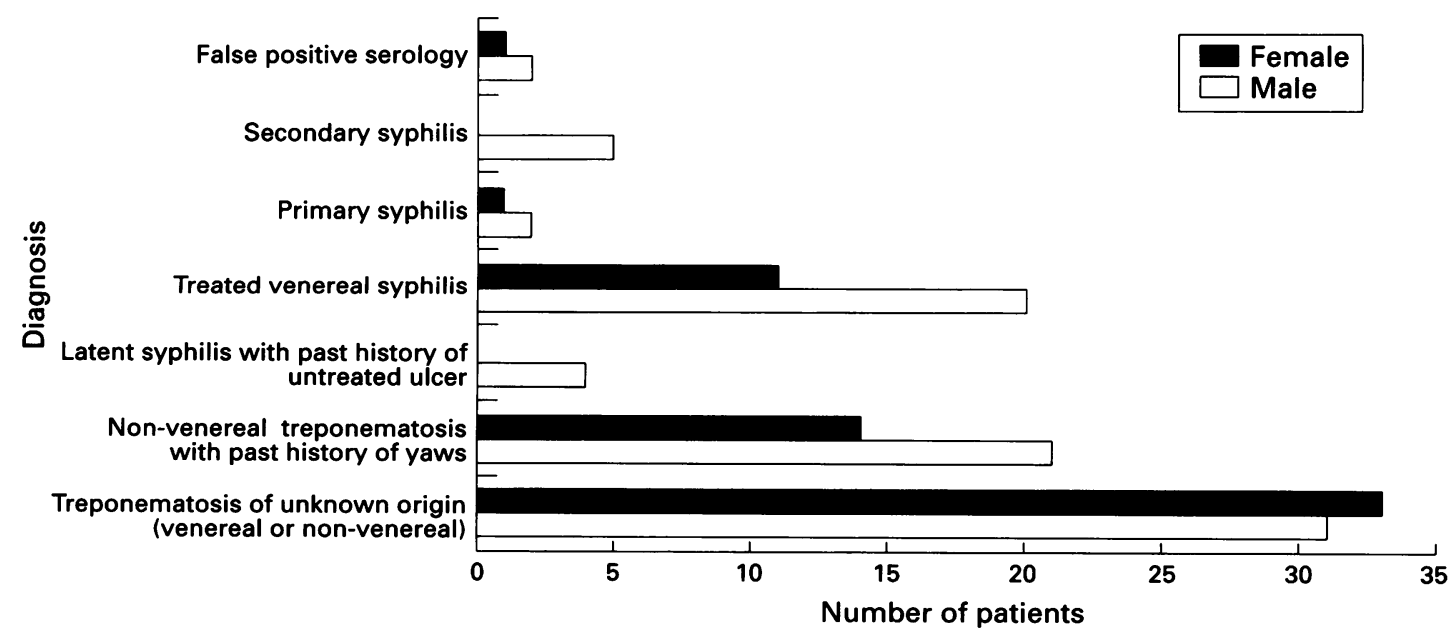


Table 2 Characteristics of patients with likely neurosyphilis

\begin{tabular}{|c|c|c|c|c|c|c|c|c|c|c|c|c|c|c|}
\hline \multirow[b]{3}{*}{$\begin{array}{l}\text { Case } \\
\text { No }\end{array}$} & \multicolumn{5}{|c|}{ Patient details } & \multirow{2}{*}{\multicolumn{4}{|c|}{ CSF analysis }} & \multirow{2}{*}{\multicolumn{3}{|c|}{ Serum analysis }} & \multirow[b]{3}{*}{ Diagnosis } & \multirow[b]{3}{*}{ Treatment } \\
\hline & \multirow[b]{2}{*}{$\begin{array}{l}\text { Agel } \\
\text { sex }\end{array}$} & \multirow{2}{*}{$\begin{array}{l}\text { Country } \\
\text { of } \\
\text { origin }\end{array}$} & \multirow{2}{*}{$\begin{array}{l}\text { Source } \\
\text { of } \\
\text { referral }\end{array}$} & \multirow[b]{2}{*}{$\begin{array}{l}\text { Clinical } \\
\text { findings }\end{array}$} & \multirow{2}{*}{$\begin{array}{l}\text { Other } \\
\text { medical } \\
\text { conditions }\end{array}$} & & & & & & & & & \\
\hline & & & & & & $\begin{array}{l}\text { Protein } \\
\text { mg/dl }\end{array}$ & $\begin{array}{l}W C C \\
\left(\times 10^{6} / l\right)\end{array}$ & $R P R$ & $F T A-A b$ & $R P R$ & $T P H A$ & $F T A-A b$ & & \\
\hline $1^{\star}$ & $74 / \mathrm{M}$ & $\begin{array}{l}\text { West } \\
\text { Indies }\end{array}$ & Psychiatry & $\begin{array}{l}\text { Dorsal } \\
\text { column } \\
\text { loss } \\
\downarrow \text { Memory }\end{array}$ & 一 & ND & ND & ND & ND & $\begin{array}{l}\text { Positive } \\
\text { Neat }\end{array}$ & Positive & Positive & $\begin{array}{l}\text { Tabes } \\
\text { dorsalis }\end{array}$ & $\begin{array}{l}\text { Lost to } \\
\text { follow up }\end{array}$ \\
\hline 2 & $62 / \mathrm{M}$ & Ethiopia & ENT & $\begin{array}{l}\text { Sensori- } \\
\text { neural } \\
\text { deafness }\end{array}$ & - & ND & ND & ND & ND & $\begin{array}{l}\text { Positive } \\
\text { Neat }\end{array}$ & Positive & Positive & $\begin{array}{l}\text { Oto- } \\
\text { syphilis }\end{array}$ & $\begin{array}{l}\text { Amoxil } \\
\text { and } \\
\text { probenecid }\end{array}$ \\
\hline 3 & $89 / \mathrm{M}$ & UK & GP & $\begin{array}{l}\text { Dorsal } \\
\text { column } \\
\text { loss }\end{array}$ & - & ND & ND & ND & ND & $\begin{array}{l}\text { Positive } \\
1: 2\end{array}$ & Positive & Positive & $\begin{array}{l}\text { Tabes } \\
\text { dorsalis }\end{array}$ & $\begin{array}{l}\text { Amoxil } \\
\text { and } \\
\text { probenecid }\end{array}$ \\
\hline $4^{\star}$ & $66 / M$ & Dominica & GP & $\begin{array}{l}\text { Unsteady } \\
\text { Dorsal } \\
\text { column } \\
\text { loss }\end{array}$ & - & 55 & 5 & NR & Positive & $\begin{array}{l}\text { Positive } \\
1: 16\end{array}$ & Positive & Positive & $\begin{array}{l}\text { Tabes } \\
\text { dorsalis }\end{array}$ & $\begin{array}{l}\text { Benzyl- } \\
\text { penicillin }\end{array}$ \\
\hline 5 & $56 / \mathrm{M}$ & Jamaica & ENT & $\begin{array}{l}\text { Sensori- } \\
\text { neural } \\
\text { deafness }\end{array}$ & - & 57 & $<1$ & NR & ND & Negative & Positive & Positive & $\begin{array}{l}\text { Oto- } \\
\text { syphilis }\end{array}$ & $\begin{array}{l}\text { Amoxil } \\
\text { and } \\
\text { probenecid }\end{array}$ \\
\hline $6^{\star}$ & $66 / \mathrm{M}$ & $\begin{array}{l}\text { West } \\
\text { Indies }\end{array}$ & PCC & $\begin{array}{l}\text { Unsteady } \\
\downarrow \text { Memory }\end{array}$ & $\uparrow \mathrm{BP}$ & 60 & 2 & Positive & Positive & $\begin{array}{l}\text { Positive } \\
1: 16\end{array}$ & Positive & Positive & $\begin{array}{l}\text { Tabes } \\
\text { dorsalis }\end{array}$ & $\begin{array}{l}\text { Benzyl- } \\
\text { penicillin }\end{array}$ \\
\hline $7^{\star}$ & $62 / M$ & Ghana & ENT & $\begin{array}{l}\text { Sensori- } \\
\text { neural } \\
\text { deafness }\end{array}$ & - & 35 & 67 & NR & NR & Negative & Positive & Positive & $\begin{array}{l}\text { Oto- } \\
\text { syphilis }\end{array}$ & $\begin{array}{l}\text { Procaine } \\
\text { penicillin }\end{array}$ \\
\hline 8 & $56 / \mathrm{M}$ & Jamaica & GP & $\begin{array}{l}\text { Sensori- } \\
\text { neural } \\
\text { deafness }\end{array}$ & - & 57 & $<1$ & NR & NR & $\begin{array}{l}\text { Positive } \\
\text { Neat }\end{array}$ & Positive & Positive & $\begin{array}{l}\text { Oto- } \\
\text { syphilis }\end{array}$ & $\begin{array}{l}\text { Amoxil } \\
\text { and } \\
\text { probenecid }\end{array}$ \\
\hline 9 & $59 / \mathrm{F}$ & Jamaica & ENT & $\begin{array}{l}\text { Sensorio- } \\
\text { neural } \\
\text { deafness }\end{array}$ & - & 58 & $<1$ & NR & NR & Negative & Positive & Positive & $\begin{array}{l}\text { Oto- } \\
\text { syphilis }\end{array}$ & $\begin{array}{l}\text { Amoxil } \\
\text { and } \\
\text { probenecid }\end{array}$ \\
\hline 10 & $81 / F$ & UK & Psychiatry & $\begin{array}{l}\text { Dorsal } \\
\text { column } \\
\text { loss }\end{array}$ & - & 35 & $<1$ & NR & NR & $\begin{array}{l}\text { Positive } \\
\text { Neat }\end{array}$ & Positive & Positive & $\begin{array}{l}\text { Tabes } \\
\text { dorsalis }\end{array}$ & $\begin{array}{l}\text { Procaine } \\
\text { penicillin }\end{array}$ \\
\hline
\end{tabular}

$\downarrow=$ reduced; $\uparrow=$ raised; $\mathrm{WCC}=$ white cell count; CSF $=$ cerebrospinal fluid; RPR $=$ rapid plasmin reagin; TPHA $=$ Treponema pallidum haemagglutination $\mathrm{FTA}-\mathrm{Ab}=$ fluorescent treponemal antibody absorption; $\mathrm{NR}=$ non-reactive; ND = not done; $\mathrm{BP}=$ blood pressure; $\star$ = history of yaws; ENT $=$ ear, nose and FTA-Ab = fluorescent treponemal antibody absorption; $\mathrm{NR}=$ non-reactive;
throat surgery; PCC = Patrick Clement's Clinic; GP = general practitioner.

Table 3 Characteristics of patients with non-syphilitic neurology

\begin{tabular}{|c|c|c|c|c|c|c|c|c|c|c|c|c|c|c|}
\hline \multirow{3}{*}{$\begin{array}{l}\text { Case } \\
\text { No }\end{array}$} & \multicolumn{5}{|c|}{ Patient details } & \multirow{2}{*}{\multicolumn{4}{|c|}{ CSF analysis }} & \multirow{2}{*}{\multicolumn{3}{|c|}{ Serum analysis }} & \multirow[b]{3}{*}{ Diagnosis } & \multirow[b]{3}{*}{ Treatment } \\
\hline & \multirow{2}{*}{$\begin{array}{l}\text { Agel } \\
\text { sex }\end{array}$} & \multirow{2}{*}{$\begin{array}{l}\text { Country } \\
\text { of } \\
\text { origin }\end{array}$} & \multirow{2}{*}{$\begin{array}{l}\text { Source } \\
\text { of } \\
\text { referral }\end{array}$} & \multirow{2}{*}{$\begin{array}{l}\text { Clinical } \\
\text { findings }\end{array}$} & \multirow{2}{*}{$\begin{array}{l}\text { Other } \\
\text { medical } \\
\text { conditions }\end{array}$} & & & & & & & & & \\
\hline & & & & & & $\begin{array}{l}\text { Protein } \\
\text { mg/dl }\end{array}$ & $\left(\times 10^{6} / \mathrm{l}\right)$ & $R P R$ & $F T A-A b$ & $R P R$ & $T P H A$ & $F T A-A b$ & & \\
\hline $1^{\star}$ & $58 / M$ & St Kitts & PCC & $\downarrow$ memory & DM & 59 & $<1$ & NR & NR & NR & Positive & Positive & $\begin{array}{l}\text { Latent NV } \\
\text { syphilis } \\
\text { DM }\end{array}$ & $\begin{array}{l}\text { Benzyl- } \\
\text { penicillin }\end{array}$ \\
\hline $2^{\star}$ & $53 / \mathrm{M}$ & Dominica & $\begin{array}{l}\text { General } \\
\text { medicine }\end{array}$ & $\begin{array}{l}\text { CVA } \\
\text { Hemi- } \\
\text { paresis }\end{array}$ & $\begin{array}{l}\text { DM } \\
\uparrow \mathrm{BP}\end{array}$ & 35 & $<1$ & NR & NR & $\begin{array}{l}\text { Positive } \\
1: 2\end{array}$ & Positive & Positive & $\begin{array}{l}\text { Latent NV } \\
\text { syphilis } \\
\text { CVA }\end{array}$ & $\begin{array}{l}\text { Lost to } \\
\text { follow up }\end{array}$ \\
\hline 3 & $69 / M$ & India & ENT & $\begin{array}{l}\text { Sensori- } \\
\text { neural } \\
\text { deafness }\end{array}$ & - & & & NR & NR & NR & Positive & Positive & $\begin{array}{l}\text { Latent V } \\
\text { syphilis } \\
\text { SNHL }\end{array}$ & $\begin{array}{l}\text { Amoxil } \\
\text { and } \\
\text { probenecid }\end{array}$ \\
\hline 4 & $55 / \mathrm{M}$ & Trinidad & GUM & $\begin{array}{l}\text { deamess } \\
\downarrow \text { memory }\end{array}$ & - & & & NR & NR & $\begin{array}{l}\text { Positive } \\
1: 8\end{array}$ & Positive & Positive & $\begin{array}{l}\text { SNHL } \\
\text { Latent V } \\
\text { syphilis } \\
\text { Dementia }\end{array}$ & $\begin{array}{l}\text { probenecid } \\
\text { None }\end{array}$ \\
\hline 5 & $53 / \mathrm{M}$ & Dominica & $\begin{array}{l}\text { General } \\
\text { medicine }\end{array}$ & $\begin{array}{l}\text { CVA } \\
\text { Hemi- } \\
\text { plegia }\end{array}$ & $\begin{array}{l}\mathrm{DM} \\
\uparrow \mathrm{BP}\end{array}$ & 35 & $<1$ & NR & NR & $\begin{array}{l}\text { Positive } \\
1: 2\end{array}$ & Positive & Positive & $\begin{array}{l}\text { Latent NV } \\
\text { syphilis } \\
\text { CVA }\end{array}$ & $\begin{array}{l}\text { Lost to } \\
\text { follow up }\end{array}$ \\
\hline $6^{\star}$ & $56 / M$ & $\begin{array}{l}\text { West } \\
\text { Indies }\end{array}$ & GP & $\begin{array}{l}\text { General } \\
\text { seizure }\end{array}$ & $\uparrow \mathrm{BP}$ & 35 & $<1$ & NR & NR & $\begin{array}{l}\text { Positive } \\
\text { Neat }\end{array}$ & Positive & Positive & $\begin{array}{l}\text { Latent NV } \\
\text { syphilis } \\
\text { CVA }\end{array}$ & $\begin{array}{l}\text { Benzathine } \\
\text { penicillin }\end{array}$ \\
\hline 7 & $67 / \mathrm{F}$ & Jamaica & ENT & Vertigo & - & 50 & $<1$ & NR & NR & NR & Positive & Positive & $\begin{array}{l}\text { Latent NV } \\
\text { syphilis } \\
\text { Ménière's } \\
\text { disease }\end{array}$ & $\begin{array}{l}\text { Benzathine } \\
\text { penicillin }\end{array}$ \\
\hline $8^{\star}$ & $65 / F$ & Jamaica & ENT & $\begin{array}{l}\text { Sensori- } \\
\text { neural } \\
\text { deafness }\end{array}$ & - & 35 & 2 & NR & NR & $\begin{array}{l}\text { Positive } \\
1: 2\end{array}$ & Positive & Positive & $\begin{array}{l}\text { Latent NV } \\
\text { syphilis } \\
\text { SNHL }\end{array}$ & $\begin{array}{l}\text { Procaine } \\
\text { penicillin }\end{array}$ \\
\hline 9 & $75 / F$ & UK & Psychiatry & $\begin{array}{l}\text { Dis- } \\
\text { orientation }\end{array}$ & - & & $<1$ & NR & NR & $\begin{array}{l}\text { Positive } \\
\text { Neat }\end{array}$ & Positive & Positive & $\begin{array}{l}\text { Latent V } \\
\text { syphilis } \\
\text { Dementia }\end{array}$ & NA \\
\hline $10^{\star}$ & $52 / \mathrm{F}$ & $\begin{array}{l}\text { West } \\
\text { Indies }\end{array}$ & PCC & $\begin{array}{l}\text { Memory } \\
\text { Loss }\end{array}$ & - & 30 & 1 & NR & NR & & & & $\begin{array}{l}\text { Latent NV } \\
\text { syphilis } \\
\text { Dementia }\end{array}$ & $\begin{array}{l}\text { Doxy- } \\
\text { cycline }\end{array}$ \\
\hline
\end{tabular}

$\downarrow=$ reduced; $\uparrow=$ raised; $\mathrm{WCC}=$ white cell count; $\mathrm{CSF}=$ cerebrospinal fluid; $\mathrm{RPR}=$ rapid plasmin reagin; TPHA $=$ Treponema pallidum haemagglutination FTA-Ab = fluorescent treponemal antibody absorption; NR = non-reactive; ND = not done; $B P=$ blood pressure; $\star$ = history of yaws; ENT = ear, nose and throat surgery; GUM = another genitourinary medicine clinic; PCC = Patrick Clement's Clinic; $\mathrm{GP}=$ general practitioner; NV = non-venereal; V = venereal; $\mathrm{NA}=$ not available.

seven patients were of Afro-Caribbean origin; one gave a history of yaws and five had scars consistent with yaws. Two patients gave a history of treated venereal syphilis and one a history of untreated latent venereal syphilis with coincidental neurological disease comprising sensorineural hearing loss (case 3) and dementia (case 4 and 9).

Of the seven patients without CSF exami- nation (Table 4), four were diagnosed with previous venereal syphilis, and coincidental multi-infarct dementia (cases 1 and 4), Alzheimer's disease (case 3), and mild memory loss (case 5). Three had latent non-venereal syphilis and concomitant cerebrovascular disease (case 6) and dementia (case 2 and 7). In the absence of CSF findings, five of these patients received adequate neurosyphilitic 
Table 4 Characteristics of patients without CSF evaluation and non-syphilitic neurology

\begin{tabular}{|c|c|c|c|c|c|c|c|c|c|c|}
\hline \multirow{3}{*}{$\begin{array}{l}\text { Case } \\
\text { No }\end{array}$} & \multicolumn{5}{|c|}{ Patient details } & & & & \multirow[b]{3}{*}{ Diagnosis } & \multirow[b]{3}{*}{ Treatment } \\
\hline & \multirow{2}{*}{$\begin{array}{l}\text { Agel } \\
\text { sex }\end{array}$} & \multirow{2}{*}{$\begin{array}{l}\text { Country } \\
\text { of } \\
\text { origin }\end{array}$} & \multirow{2}{*}{$\begin{array}{l}\text { Source } \\
\text { of } \\
\text { referral }\end{array}$} & \multirow{2}{*}{$\begin{array}{l}\text { Clinical } \\
\text { findings }\end{array}$} & \multirow{2}{*}{$\begin{array}{l}\text { Other } \\
\text { medical } \\
\text { conditions }\end{array}$} & \multicolumn{3}{|c|}{ Serum analysis } & & \\
\hline & & & & & & $R P R$ & $T P H A$ & $F T A-A b$ & & \\
\hline $1^{\star}$ & $77 / M$ & Dominica & GP & $\downarrow$ memory & $\begin{array}{l}\text { IHD } \\
\text { DM }\end{array}$ & $\begin{array}{l}\text { Positive } \\
1: 8\end{array}$ & Positive & Positive & $\begin{array}{l}\text { Treated V } \\
\text { syphilis } \\
\text { Multi-infarct } \\
\text { dementia }\end{array}$ & None \\
\hline 2 & $71 / F$ & Jamaica & ENT & $\begin{array}{l}\text { Sensori- } \\
\text { neural } \\
\text { deafness }\end{array}$ & $\uparrow \mathrm{BP}$ & $\begin{array}{l}\text { Positive } \\
\text { neat }\end{array}$ & Positive & Positive & $\begin{array}{l}\text { Latent NV } \\
\text { syphilis } \\
\text { Dementia }\end{array}$ & $\begin{array}{l}\text { Amoxil } \\
\text { and } \\
\text { probenecid }\end{array}$ \\
\hline 3 & $84 / F$ & UK & PCC & $\downarrow$ memory & PVD & $\begin{array}{l}\text { Positive } \\
\text { neat }\end{array}$ & Positive & Positive & $\begin{array}{l}\text { Treated V } \\
\text { syphilis } \\
\text { Alzheimer's } \\
\text { dementia }\end{array}$ & $\begin{array}{l}\text { Amoxil } \\
\text { and } \\
\text { probenecid }\end{array}$ \\
\hline 4 & $78 / \mathrm{F}$ & UK & PCC & $\downarrow$ memory & - & $\begin{array}{l}\text { Positive } \\
\text { neat }\end{array}$ & Positive & Positive & $\begin{array}{l}\text { dementala } \\
\text { Latent V } \\
\text { syphilis } \\
\text { Multi-infarct } \\
\text { dementia }\end{array}$ & $\begin{array}{l}\text { Amoxil } \\
\text { and } \\
\text { probenecid }\end{array}$ \\
\hline 5 & $38 / F$ & Zimbabwe & GP & $\downarrow$ memory & - & NR & Positive & Positive & $\begin{array}{l}\text { Treated V } \\
\text { syphilis }\end{array}$ & None \\
\hline 6 & $60 / \mathrm{F}$ & $\begin{array}{l}\text { West } \\
\text { Indies }\end{array}$ & PCC & CVA & - & NR & Positive & Positive & $\begin{array}{l}\text { Latent NV } \\
\text { syphilis }\end{array}$ & $\begin{array}{l}\text { Procaine } \\
\text { penicillin }\end{array}$ \\
\hline 7 & $67 / F$ & Jamaica & GP & $\downarrow$ memory & - & NR & Positive & Positive & $\begin{array}{l}\text { Latent NV } \\
\text { syphilis } \\
\text { Dementia }\end{array}$ & Doxycycline \\
\hline
\end{tabular}

$\downarrow=$ reduced; $\uparrow=$ raised; $R P R=$ rapid plasmin reagin; TPHA = Treponema pallidum haemagglutination; FTA-Ab = fluorescent treponemal antibody absorption; NR $=$ non-reactive; $\mathrm{BP}=$ blood pressure; CVA $=$ cardiovascular accident; $\mathrm{PVD}=$ peripheral vascular disease; $\star$ history of yaws; ENT $=$ ear, nose and throat surgery; PCC = Patrick Clement's Clinic; GP = general practitioner; $\mathrm{NV}=$ non-venereal; $\mathrm{V}=$ venereal.

treatment and two with documentation of previous adequate treatment did not (cases 1 and 5).

In accordance with current clinic protocol those patients without a history of penicillin allergy were treated with the oral amoxicillin $3 \mathrm{~g}$ and oral probenecid $1 \mathrm{~g}$ twice daily for 14 or 21 days, intramuscular procaine penicillin $600000 \mathrm{IU} /$ day for 21 days, or intravenous benzylpenicillin $2.4 \mathrm{~g}$ daily for 14 days. In cases of penicillin allergy oral doxycycline 300 mg daily for 21 days was prescribed. No patient received steroid therapy. Latent nonvenereal syphilis was treated with $2.4 \mathrm{mU}$ benzathine penicillin on days 1 and 7 .

\section{Discussion}

Possibly all patients infected with Treponema pallidum harbour the spirochaete in the central nervous system after primary infection. ${ }^{15}$ Between $7 \%$ and $9 \%$ of untreated syphilitic patients develop a symptomatic neurosyphilitic syndrome. ${ }^{17-19}$ Asymptomatic neurosyphilis with abnormal CSF findings is likely to progress to active neurosyphilis. ${ }^{20}$ The clinical features of the 10 patients in this cohort diagnosed with neurosyphilis included five with sensorineural hearing loss and five with features of tabes dorsalis. Adequate antibiotic therapy can cure central nervous system disease when neurological disease occurs early (associated with secondary syphilis). In late tertiary disease antibiotics may prevent further damage from neurosyphilis but do not repair damage that has already occurred. In some cases tertiary neurosyphilis progresses despite adequate therapy..$^{21}$ It is important, therefore, that patients with neurological disorders and positive treponemal serology are diagnosed accurately.

The multiethnicity of the patient population requires a sound knowledge of the prevalence of the non-venereal treponemal diseases throughout the world; only $12 \%$ of the cohort were Western European. The diagnosis of non-venereal treponematoses must be considered in the appropriate clinical and historical setting. ${ }^{22}$ Identifying a past history of yaws or venereal syphilis is not always possible and the final diagnosis may be one of exclusion. The WHO and United Nations Children's Fund (UNICEF) supported mass treatment campaigns for the eradication of yaws from 1954 to 1963 , but lack of public health measures has led to a recent resurgence in many tropical countries. ${ }^{23}$ Seroprevalence rates vary between $15 \%$ and $95 \% .^{24}$ Endemic syphilis is now only found among the semi-nomads of the Arabian peninsula and Saharan Africa and there are no data regarding the current worldwide prevalence of active pinta. ${ }^{22}$

A seropositive patient with neurological problems does not necessarily have to be treated for neurosyphilis if a lumbar puncture can be performed to exclude it. However, when patients decline the procedure it is better to err on the side of caution and treat a potentially treatable condition. The indolent course of neurosyphilis also raises the question of to what lengths one searches for signs of nervous system involvement in latent venereal syphilis. Should all patients with latent syphilis undergo CSF examination? The current clinic policy is to perform lumbar puncture in only those cases with positive neurological findings, with clinical evidence of cardiovascular syphilis or with an elevated RPR. In this cohort CSF parameters were not known for 3/10 patients with suspected neurosyphilis and $7 / 17$ patients with neurological disease not thought to be due to syphilis. While one patient was lost to follow up, the remaining nine patients received a standard neurosyphilitic antibiotic regimen. Obtaining consent in patients with latent syphilis, without neurological symptoms, may give rise to an even higher rate of refusal although detecting and adequately treating asymptomatic neurosyphilis can prevent its progression to symptomatic disease. 
In the diagnosis of neurosyphilis the CSFFTA ABS test appears more sensitive for screening but is less specific than the CSF $R P R$ test in distinguishing active neurosyphilis from past neurosyphilis. False positive tests may occur; indeed some cases of neurosyphilis lack a reactive CSF RPR. ${ }^{1425}$ Clinical judgment is still essential in establishing the diagnosis. The intrathecal synthesis of IgG antibody to Treponema pallidum occurs in neurosyphilis, and oligoclonal bands in the CSF have been recognised in at least $75 \%$ of cases. ${ }^{26}$ Further experience with this test may provide an additional investigative tool to distinguish active from asymptomatic syphilis. Selecting patients presenting with latent syphilis for CSF examination, to exclude asymptomatic neurosyphilis, may be improved by serological testing with EIA, Captia Syphilis-G. ${ }^{27}$ Detection of a positive EIA, in a patient with a negative RPR or VDRL and history of treatment, could help assess the efficacy of the previous treatment and the need for more intensive investigations. ${ }^{28}$

The classic clinical syndrome of neurosyphilis is now rarely seen. Clinical improvement or the arrest of clinical disease following treatment was not seen in our series. Is neurosyphilis almost dead and gone? The coincidental use of treponemicidal antibiotics in the past 50 years - that is, penicillin, cephalosporins, tetracyclines, macrolides, and others, in the course of an individuals lifetime, has probably impacted on the clinical manifestation and course of neurosyphilis. The early diagnosis and adequate treatment of infectious syphilis has also greatly diminished the incidence of neurosyphilis. The use of benzathine penicillin, which does not penetrate the CSF, in the treatment of infectious syphilis will have a bearing on the diagnosis of neurosyphilis. In this series the high number of patients from areas of high endemicity for endemic nonvenereal treponematoses further complicates accurate diagnosis.

Establishing or excluding a diagnosis of neurosyphilis can be troublesome in patients also infected with the human immunodeficiency virus (HIV) in whom pleocytosis and raised protein content are more common. ${ }^{29}$ Of particular importance are the patients at risk from AIDS, with recent reported cases of the development of neurosyphilis in HIV positive patients despite adequate treatment for early syphilis. ${ }^{30}$ The three HIV positive patients in this cohort were diagnosed with latent venereal syphilis and did not undergo CSF examination for their positive treponemal serology. The hypothesis that HIV infection may modify syphilitic infection has not been supported by necropsy findings in tertiary syphilis. ${ }^{31}$ However, such patients may need to be monitored more closely. ${ }^{32}$

In summary, the diagnosis of CNS involvement in patients with positive treponemal serology depends on historical findings and clinical judgment, supported by serological and CSF analysis. A well defined clinic protocol will ensure that patients are appropriately managed and adequately treated, and that incidence and follow up are recorded.

1 Adler MW. Epidemiology of sexually transmitted diseases In: Sexually transmitted diseases. London: Csonka GW and Oates, 1990:6-16

2 Summary information from form KC60 1993-1994. London: Department of Health Statistics Division.

3 Linglof T. Rapid increase of syphilis and gonorrhoea in parts of the former USSR. Sex Transm Dis 1995;22: $160-1$

4 World Health Organisation. Treponemal infections. Report of a WHO scientific group. Geneva: World Health Organisation Technical Report Series 674, 1982

5 Johnson RA, White M. Syphilis in the 1990s: cutaneous and neurological manifestations. Sem Neurol 1992;12. 287-98.

6 Ashcroft MT, Miall WE, Standard KL, Urquhart AE. Serological tests for treponemal disease in two Jamaican countries. Br $\mathcal{F}$ Vener Dis 1967;43:96-104.

7 Hardy PH. Prospects for improved laboratory diagnoses of treponemal infections and species differentiation. Rev Infect Dis 1985;7:300-3.

8 Swartz M. Neurosyphilis. In: Holmes KK, March PA Sparling PF, Weisner PS, eds. Sexually transmitted disease. New York: McGraw-Hill, 1984:318-34.

9 Csonka GW. Syphilis. In: Weatherall DJ, Ledingham JGG Warrell DA, eds. Oxford textbook of medicine. 2nd ed. Oxford: Oxford University Press, 1987;1:5·386-5.403.

10 Perdup A. Late syphilis, with particular reference to clinica manifestations, serology and treatment. Geneva: WHO 1980:INT/VDT/ 80.371.

11 Centers for Disease Control. STD treatment guidelines. $M M W R$ 1993;34:1-120.

12 Felman YM. Lumbar puncture in asymptomatic neurosyphilis. Arch Intern Med 1985;145:422-3.

13 Hooshmand $\mathrm{H}$, Escobar MR, Kopf SW. Neurosyphilis. A study of 241 patients. $\mathscr{f} A M A 1972 ; 210: 221-5$

14 Jaffe HW, Larsen SA, Peters M, Jove DF, Lopez B, Schroeter AL. Tests for treponemal antibodies in CSF. Arch Intern Med 1978;138:252-5.

15 Harris A, Bossak HM, Deacon WE, Bunch WL. Com parison of fluorescent antibody absorption tests with other tests for syphilis on CSF. Br f Vener Dis 1960;36. 178-80.

16 The Brent Profile 1994. Brent Council.

17 Wolters EC, Hische EA, Tutuarima JA, van Trotsenbur L, van Eijk RV, Bos JD, et al. Central nervous system involvement in early and late syphilis: the problem of invelomatic neurosyphilis. 7 Neur $S c i 1988 ; 88.229-39$.

18 Gistand T The Oslo study of untreated patients Acta Dermatol Venereol 1955;35:S34:1-368.

19 Kofman O. The changing pattern of neurosyphilis Can Med Ass F 1956;74:807-12.

20 Moore JEE, Hopkins HH. Asymptomatic neurosyphilis the prognosis of early and late asymptomatic neurosyphilis. $\mathcal{F} A M A$ 1980;95:1937-4

21 Yoder FW. Penicillin treatment of neurosyphilis: are recommended dosages sufficient? $\mathcal{F} A M A$ 1973;232:270-75.

22 Koff AB, Rosen T. Nonvenereal treponematoses: yaws, endemic syphilis and pinta. $\mathcal{F}$ Am Acad Dermatol 1993;29. 519-35.

23 Perine PL, Hopkins DR, Nierne PUA, St John RK, Causse G, Antal GM. Yaws, endemic syphilis and pinta. In Handbook of endemic treponematoses. Geneva: World Health Organisation, 1984:1-53.

24 Nagren DS. Yaws. Cutis 1986:303-5.

25 Luger A, Schmidt BL, Steyrer K, Schonwald E. Diagnosis of neurosyphilis by examination of the CSF. BrF Vener Dis 1981;57:232-7.

26 Dari L, Schmidt J. Clinical significance of cerebrospinal fluid tests for neurosyphilis. Ann Neurol 1989;25:50-5.

27 Prange HW, Moskophidis M, Schipper H, Muller F. Relationship between neurological features and intrathecal synthesis of IgG antibodies to Treponema pallidum in untreated and treated human neurosyphilis. $f$ Neurol 1983;230:241-52.

28 Young $\mathrm{H}$, Moyes A, McMillan A, Robertson DH. Screening for treponemal infection by a new enzym imeening for treponemal infection by a

29 Appleman ME, Marshall DW, Brey RI, Houk RW, Beatty DC, Winn RE, et al. Cerebrospinal fluid abnormalities in patients without AIDS who are seropositive for HIV. $f$ patients without AIDS who are seropositive for HIV. Infect Dis 1988;158:193-9.

30 Johns DR, Tierney M, Felsenstein D. Alteration in the nat ural history of neurosyphilis by concurrent infcetion with HIV. N Engl f Med 1987;316:1569-672.

31 Musher MD. Syphilis, neurosyphilis, penicillin and AIDS. 7 Infect Dis 1991;163:1201-6.

32 Terry PM, Page ML, Goldmeier D. Are serological tests of value in diagnosis and monitoring response to treatment of syphilis in patients infected with HIV? Genitourin Med 1988;64:219-22. 